\title{
Decentralising diabetes care from hospitals to primary health care centres in Malawi
}

\author{
Colin Pfaff', Gift Malamula ${ }^{1}$, Gabriel Kamowatimwa ${ }^{1}$, Jo Theu ${ }^{1}$, Theresa J Allain ${ }^{2}$, Alemayehu \\ Amberbir ${ }^{1,3}$, Sunganani Kwilasi ${ }^{1}$, Saulos Nyirenda ${ }^{4}$, Martias Joshua ${ }^{4}$, Jane Mallewa ${ }^{2}$, Clement \\ Mandala $^{5}$, Joep J van Oosterhout ${ }^{1,2}$, Monique van Lettow ${ }^{1,3}$
}

1. Dignitas International, Zomba, Malawi

2. Department of Medicine, University of Malawi College of Medicine, Blantyre, Malawi/Kamuzu University of Health Sciences

3. Dalla Lana School of Public Health, University of Toronto, Toronto, Canada

4. Ministry of Health, Zomba, Malawi

5. Diabetes Association of Malawi, Blantyre, Malawi.

Correspondence: Colin Pfaff; (colinpfaff@yahoo.co.uk)

\section{Background}

\section{Abstract}

Non-communicable diseases (NCDs) such as diabetes and hypertension have become a prominent public health concern in Malawi, where health care services for NCDs are generally restricted to urban centres and district hospitals, while the vast majority of Malawians live in rural settings. Whether similar quality of diabetes care can be delivered at health centres compared to hospitals is not known.

Methods

We implemented a pilot project of decentralized diabetes care at eight health centres in four districts in Malawi. We described differences between district hospitals and rural health centres in terms of patient characteristics, diabetes complications, cardiovascular risk factors, and aspects of the quality of care and used multivariate logistic regression to explore factors associated with adequate diabetes and blood pressure control.

Results

By March 2019, 1339 patients with diabetes were registered of whom 286 (21\%) received care at peripheral health centres. The median duration of care of patients in the diabetes clinics during the study period was 8.8 months. Overall, HIV testing coverage was $93.6 \%$, blood pressure was recorded in $92.4 \%$; $68.5 \%$ underwent foot examination of whom $35.0 \%$ had diabetic complications; $30.1 \%$ underwent fundoscopy of whom $15.6 \%$ had signs of diabetic retinopathy. No significant differences in coverage of testing for diabetes complications were observed between health facility types. Neither did we find significant differences in retention in care (72.1 vs. $77.6 \%$; $=0.06)$, adequate diabetes control (35.0\% vs. $37.8 \%$; $=0.41)$ and adequate blood pressure control $(51.3 \%$ vs. $49.8 \%$; $\mathrm{p}=0.66)$ between hospitals and health centres. In multivariate analysis, male sex was associated with adequate diabetes control, while lower age and normal body mass index were associated with adequate blood pressure control; health facility type was not associated with either.

Conclusion

Quality of care did not appear to differ between hospitals and health centres, but was insufficient at both levels.

Keywords: Diabetes care, decentralization, non-communicable diseases, Malawi, health centres.

\section{Introduction}

In sub-Saharan African countries, non-communicable diseases (NCDs) such as diabetes and hypertension have become an increasing public health concern and are expected to overtake HIV/AIDS as the leading cause of death in $2030^{1}$. A recent large population-based study in rural and urban Malawi found a prevalence of hypertension of $14.7 \%$ and $13.6 \%$, and a prevalence of diabetes of $3.0 \%$ and $1.7 \%$, respectively ${ }^{2}$. Specialized health care services to manage NCDs are mostly situated in urban centres and hospitals, while the vast majority of Malawians live in rural settings. Rural health centres in Malawi have limited capacity to manage NCDs, especially diabetes. A study in health centres near Lilongwe found that staff lacked knowledge regarding diabetes diagnosis and only $20 \%$ of facilities had a glucometer and regular supplies of diabetes drugs ${ }^{3}$. In a similar study in 32 health centres in Northern Malawi, 32\% of facilities had a glucometer and none had an uninterrupted supply of diabetic medication ${ }^{4}$. A study in 55 health centres in all three regions found that $38 \%$ of health centres had glucometers, 24\% had urine glucose dipsticks and only $4 \%$ had first-line medicines for treatment of diabetes ${ }^{5}$.

As structured NCD care has not been implemented widely in Malawi at local health centres, we describe a process of decentralizing diabetes care from district hospital to health centre level in four rural districts. After an initial 12 months of implementation, we describe differences between hospitals and health centres in terms of patient characteristics, diabetes and hypertension related complications, cardiovascular disease risk, and the quality of care provided for patients with diabetes. In addition, we explored factors associated with adequate diabetes control and with adequate blood pressure (BP) control.

\section{Background}

Health centres provide the first point of contact for most patients in the Malawi health system. Patients needing more advanced care are referred to a district hospital, from where further referral to one of four central urban hospitals is 
possible. Prior to the pilot, diabetes care was only available at central and district hospitals in Malawi. Some health centres were able to diagnose diabetes, however for treatment, patients were referred to the district hospital. BP measurement was generally available at health centres but often only one anti-hypertensive drug was available and trained staff and equipment were limited. Screening for complications such as diabetic retinopathy was only conducted at district hospitals, and rates of screening were inconsistent. If retinopathy was detected, patients needed referral to a central hospital for laser treatment, although this happened inconsistently.

As $84 \%$ of the population of Malawi reside in rural areas far from district hospitals, the poor availability of diabetes treatment at health centres forced patients to travel long distances each month to receive care. Prior to the pilot we conducted an informal survey of diabetes patients receiving care at one district hospital by examining their health records and mapping their location of residence. This exercise revealed that $25 \%$ of patients travelled $80 \mathrm{~km}$ or more to receive standard diabetic care.

\section{Methodology}

From 1 April 2018 to 31 March 2019 we implemented a pilot project of decentralized care for patients with diabetes at eight health centres in four districts (Zomba, Phalombe, Machinga and Mulanje). Patients already in care in the diabetes clinics in the district hospitals were offered the opportunity to transfer their care to a health centre. Those newly diagnosed with diabetes either at the hospital or health centre were offered to continue their care at hospital or health centre level. Patients could make this choice at any time, but were encouraged to continue in care at the facility they had chosen.

Nurses and medical assistants (mid-level clinicians with 2 years of training) underwent three days training in diabetes diagnosis and management including screening for complications. The training was followed by two- weekly visits of a clinical-officer mentor (mid-level clinician with 3 years of training). This mentor worked closely with district pharmacists to establish systems of regular drug supply. At each facility an Expert Diabetic Client (EDC), a patient willing to disclose their diabetes diagnosis, known to have excellent adherence to life-style measures and medications and having good diabetic control, gave health education sessions at the health facilities and provided home visit support to patients with poor diabetic control. EDCs were members of the Diabetic Association of Malawi (DAM) and acted as the link between the DAM and patients at the facility level. Systems were developed to ensure patients requiring treatment at central hospital levels (e.g. laser treatment for diabetic retinopathy) were identified and referred as needed.

\section{Data collection and statistical analysis}

All diabetes clinics utilized a "NCD master-card" as a facility-based patient record. These standardized Ministry of Health tools were derived from the widely used antiretroviral therapy (ART) master-cards from the HIV program. Data from the NCD master-cards were captured electronically by a project roving data collection officer at the end of each clinic.

Classification of diabetes type 1 and type 2 was made on an individual basis using age of onset, requirement of insulin, Body Mass Index (BMI), duration of symptoms and nature of hospital admissions as criteria.
Urine was checked using urine dipstix and "proteinuria" was defined as any protein (trace to 3+). Retinal screening was performed by clinical officers trained in fundoscopy using the direct method with hand-held opthalmoscopes through a dilated pupil. At Zomba Central Hospital, a binocular indirect method was also used, using a combination of a slit lamp and 90D Volk lens. Retinopathy was defined as the presence of any retinal changes associated with diabetes including nonproliferative and proliferative changes. BP was measured by an automated measuring device (Cradle VSA Microlife blood pressure monitor). Glucose level was determined by pointof-care glucometers (SD Check). Glycosylated haemoglobin (HbA1C) testing was not available at the facilities involved in this pilot.

Adequate glucose control was defined as fasting blood glucose $(\mathrm{FBG})<130 \mathrm{mg} / \mathrm{dL}$ at the last visit. Adequate blood pressure (BP) control was defined as systolic $<140$ and diastolic $<90 \mathrm{mmHg}$ at the last visit. Quality of care was measured by completeness of measurements (BP, weight, FBG) and screening for feet abnormalities, proteinuria and retinopathy performed at clinic visits and documented in the NCD master-cards. Retention in care was defined as those ever registered who made a visit in the last quarter of the project.

\section{Analysis}

Descriptive statistics were used to characterize study participants who attended the NCD clinics in the hospitals and rural health centres and to describe the quality of care indicators. Characteristics were described with numbers and proportions or medians with interquartile ranges (IQR). Comparisons between groups were made using chi-square tests for categorical variables and non-parametric tests for medians. Missing data were treated as additional categories.

Multivariable binary logistic regression analysis was used to identify factors associated with adequate diabetes control and with adequate BP control. Univariate odds ratios (OR) with $95 \% \mathrm{CI}$ were calculated for each variable in the model using normal approximation methods. Adjusted OR (aOR) with 95\% confidence intervals (CI) were calculated for each model after adjustment for health facility type, age, gender, diabetes type, duration in care, BMI and HIV status. All variables were simultaneously entered in the logistic regression model and tested for removal through backward stepwise selection. A 0.05 significance level was set for statistical testing. Analyses were conducted using IBM SPSS Statistics 26 (IBM, Armonk, NY, USA).

\section{Ethical considerations}

As a retrospective audit of routinely collected, standard service delivery data that had been fully anonymized before analysis, we were exempted by the College of Medicine Research and Ethics Committee (P.09/18/2470) from obtaining individual informed consent. Before the implementation of the pilot project of decentralized diabetes care, we had obtained support from district health offices.

\section{Results}

Demographics, comorbidities and risk factors at enrolment (Table 1)

By the end of the pilot (31 March 2019) 1339 patients with diabetes had been registered of whom $21 \%$ received care at peripheral health centres. Of all patients, $59.3 \%$ were female and the median age was 53 years (IQR 41-63); the median 
Table 1. Patient Characteristics at Baseline (first visit)

\begin{tabular}{|c|c|c|c|c|}
\hline Number of patients & $\begin{array}{l}\text { Overall } \\
1339\end{array}$ & $\begin{array}{c}\text { District } \\
\text { Hospital } \\
1053\end{array}$ & $\begin{array}{l}\text { Health Centre } \\
\qquad 286\end{array}$ & $p$-value* \\
\hline Age, median (IQR) & $\begin{array}{l}53(41- \\
63)\end{array}$ & $53(41-63)$ & $53(41-63)$ & 0.84 \\
\hline missing, $\mathrm{n}(\%)$ & $85(6.3)$ & $61(5.8)$ & $24(8.4)$ & \\
\hline \multicolumn{5}{|l|}{ Sex } \\
\hline Male, n (\%) & $\begin{array}{c}545 \\
(40.7)\end{array}$ & 441 (41.9) & $104(36.4)$ & 0.10 \\
\hline Female, n (\%) & $\begin{array}{c}794 \\
(59.3)\end{array}$ & $612(58.1)$ & $182(63.6)$ & \\
\hline \multicolumn{5}{|l|}{ Diabetes Type } \\
\hline Type 1; n (\%) & $\begin{array}{c}155 \\
(11.6)\end{array}$ & $131(12.5)$ & $24(8.4)$ & 0.06 \\
\hline Type 2; n (\%) & $\begin{array}{c}1180 \\
(88.1)\end{array}$ & 918 (87.5) & $262(91.6)$ & \\
\hline missing, $\mathrm{n}(\%)$ & $4(0.3)$ & $4(0.4)$ & 0 & \\
\hline \multicolumn{5}{|l|}{ HIV status } \\
\hline Unknown & $86(6.4)$ & $65(6.2)$ & $21(7.3)$ & 0.71 \\
\hline Positive & $51(3.8)$ & $39(3.7)$ & $12(4.2)$ & \\
\hline Negative & $\begin{array}{c}1202 \\
(89.8)\end{array}$ & $949(90.1)$ & $253(88.5)$ & \\
\hline \multicolumn{5}{|l|}{$\begin{array}{l}\text { ART status among those } \\
\text { HIV+ }\end{array}$} \\
\hline On ART & $44(86.3)$ & $34(87.2)$ & $10(83.3)$ & 0.74 \\
\hline unknown & $7(13.7)$ & $5(12.8)$ & $2(16.7)$ & \\
\hline \multicolumn{5}{|l|}{ BMI } \\
\hline Median (IQR) & $\begin{array}{l}25.3 \\
(21.5- \\
29.8)\end{array}$ & $\begin{array}{l}25.5(21.6- \\
\quad 30.1)\end{array}$ & $24.8(21.0-29.2)$ & 0.20 \\
\hline Underweight < 18.5 & $106(7.9)$ & $75(7.1)$ & $31(10.8)$ & 0.01 \\
\hline $\begin{array}{l}\text { Normal weight } 18.5 \text { to } \\
24.9\end{array}$ & $\begin{array}{c}378 \\
(28.2)\end{array}$ & $288(27.4)$ & $90(31.5)$ & \\
\hline Overweight 25 to 29.9 & $\begin{array}{l}282 \\
(21.1)\end{array}$ & 216 (20.5) & $66(23.1)$ & \\
\hline Obese $>=30$ & $\begin{array}{c}251 \\
(18.7)\end{array}$ & $200(19.0)$ & $51(17.8)$ & \\
\hline Missing, $\mathrm{n}(\%)$ & $\begin{array}{c}322 \\
(24.0)\end{array}$ & $274(26.0)$ & $48(16.8)$ & \\
\hline \multicolumn{5}{|l|}{ Hypertension Status } \\
\hline $\begin{array}{l}\text { Has diagnosis of } \\
\text { Hypertension }\end{array}$ & $\begin{array}{c}720 \\
(53.8)\end{array}$ & $552(52.4)$ & $168(58.7)$ & 0.14 \\
\hline $\begin{array}{l}\text { No diagnosis of } \\
\text { hypertension }\end{array}$ & $\begin{array}{c}541 \\
(40.4)\end{array}$ & $436(41.4)$ & $105(36.7)$ & \\
\hline Missing, $\mathrm{n}(\%)$ & $78(5.8)$ & $65(6.2)$ & $13(4.5)$ & \\
\hline \multicolumn{5}{|l|}{$\begin{array}{l}\text { Duration of hypertension } \\
\text { (months from diagnosis) }\end{array}$} \\
\hline Months, median (IQR) & $25(7-82)$ & $25(8-86)$ & $25(5-64)$ & 0.92 \\
\hline Missing, $\mathrm{n}(\%)$ & $2(0.3)$ & & & \\
\hline $\begin{array}{l}\text { Median (IQR) fasting } \\
\text { blood glucose }\end{array}$ & $\begin{array}{c}180 \\
(124- \\
292)\end{array}$ & 179 (125-289) & $182(121-302)$ & 0.80 \\
\hline $\begin{array}{l}\text { Good glucose control } \\
(\leq 130)\end{array}$ & $\begin{array}{c}346 \\
(25.8)\end{array}$ & 285 (27.1) & $61(21.3)$ & 0.001 \\
\hline
\end{tabular}




\section{Table 1 Cont...}

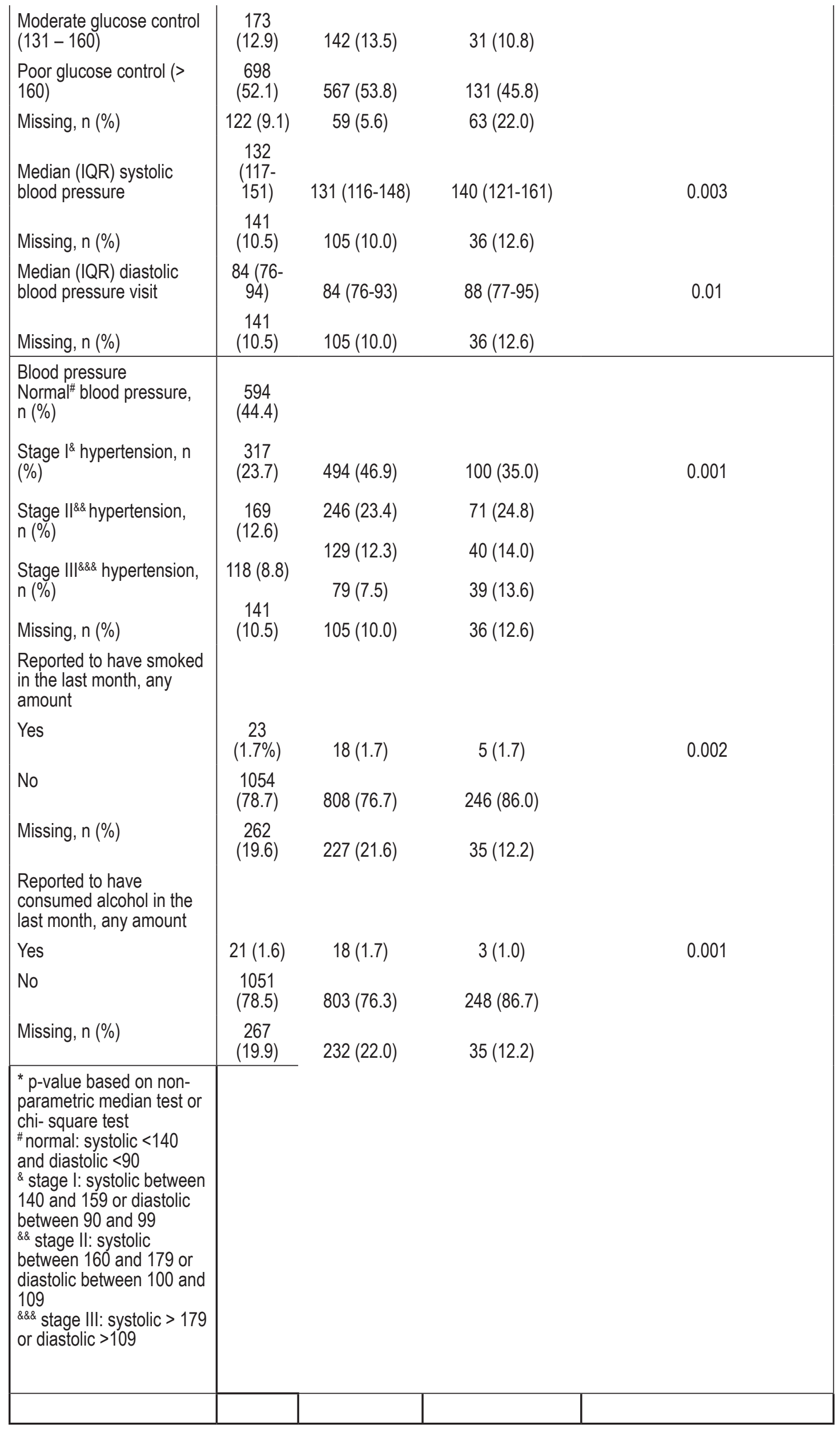


Table 2. Quality of Care by Site Status

\begin{tabular}{|c|c|c|c|c|}
\hline & Overall & $\begin{array}{l}\text { District } \\
\text { Hospital }\end{array}$ & Health Centre & $p$-value \\
\hline No. of patients & 1339 & 1053 & 286 & \\
\hline $\begin{array}{l}\text { Median number of months in } \\
\text { the program (first visit to last } \\
\text { visit) (IQR) }\end{array}$ & $\begin{array}{l}8.8(3.7- \\
11.4)\end{array}$ & $9.5(3.7-11.7)$ & $7.2(3.9-9.7)$ & 0.001 \\
\hline \multicolumn{5}{|l|}{$\begin{array}{l}\text { Retention in care - patients } \\
\text { who had a visit in last quarter } \\
\text { (1 Jan } 2019 \text { to } 31 \text { March } \\
\text { 2019) among those ever } \\
\text { registered; } n(\%)\end{array}$} \\
\hline Did not have visit & $358(26.7)$ & $294(27.9)$ & $64(22.4)$ & 0.06 \\
\hline Did have visit & 981 (73.3) & 759 (72.1) & $222(77.6)$ & \\
\hline \multicolumn{5}{|l|}{$\begin{array}{l}\text { Patients who had weight } \\
\text { recorded on master-card at } \\
\text { the last visit, } n(\%)\end{array}$} \\
\hline Not recorded & $221(16.5)$ & $178(16.9)$ & $43(15.0)$ & 0.45 \\
\hline Recorded & $\begin{array}{c}1118 \\
(83.5)\end{array}$ & $875(83.1)$ & $243(85.0)$ & \\
\hline \multicolumn{5}{|l|}{$\begin{array}{l}\text { Patients who had their blood } \\
\text { glucose recorded on master- } \\
\text { card (FBS or RBS) at the last } \\
\text { visit, } n(\%)\end{array}$} \\
\hline Not recorded & $100(7.5)$ & $44(4.2)$ & $56(19.6)$ & 0.001 \\
\hline Recorded & $\begin{array}{c}1239 \\
(92.5)\end{array}$ & $1009(95.8)$ & $230(80.4)$ & \\
\hline \multicolumn{5}{|l|}{$\begin{array}{l}\text { Patients who had their blood } \\
\text { pressure recorded on master- } \\
\text { card at the last visit, } n(\%)\end{array}$} \\
\hline Not recorded & $102(7.6)$ & $75(7.1)$ & $27(9.4)$ & 0.19 \\
\hline Recorded & $\begin{array}{l}1237 \\
(92.4)\end{array}$ & $978(92.9)$ & $259(90.6)$ & \\
\hline \multicolumn{5}{|l|}{ HIV test coverage, $n(\%)$} \\
\hline $\begin{array}{l}\text { Tested - new or previous } \\
\text { (pos or neg) }\end{array}$ & $\begin{array}{l}1253 \\
(93.6)\end{array}$ & $988(93.8)$ & $265(92.7)$ & 0.47 \\
\hline Not tested & $86(6.4)$ & $65(6.2)$ & $21(7.3)$ & \\
\hline \multicolumn{5}{|l|}{$\begin{array}{l}\text { Patients in the cohort who } \\
\text { had retinal screening done at } \\
\text { any time, } n(\%)\end{array}$} \\
\hline Never done & $936(69.9)$ & $663(63.0)$ & $273(95.5)$ & 0.001 \\
\hline Ever done & $403(30.1)$ & $390(37.0)$ & $13(4.5)$ & \\
\hline $\begin{array}{l}\text { Findings suggesting diabetic } \\
\text { retinopathy among patients } \\
\text { who received retinal } \\
\text { screening, } n / n(\%)\end{array}$ & $\begin{array}{l}63 / 403 \\
(15.6)\end{array}$ & $59 / 390(15.1)$ & $4 / 13(30.8)$ & 0.13 \\
\hline \multicolumn{5}{|l|}{$\begin{array}{l}\text { Patients who had urine } \\
\text { testing done at any time, } n / n \\
(\%)\end{array}$} \\
\hline never done & $\begin{array}{c}1327 \\
(99.1)\end{array}$ & $1051(99.8)$ & $276(96.5)$ & 0.001 \\
\hline done & $12(0.9)$ & $2(0.2)$ & $10(3.5)$ & \\
\hline $\begin{array}{l}\text { Patients with proteinura in } \\
\text { those who had urine testing, } \\
\mathrm{n} / \mathrm{n}(\%)\end{array}$ & $\begin{array}{c}4 / 12 \\
(33.3)\end{array}$ & $2 / 2(100)$ & $2 / 10(20.0)$ & 0.03 \\
\hline
\end{tabular}


Table 2 Cont...

Patients who had foot check done at the last visit, $n(\%)$

done

926 (69.2)

695 (66.0)

$231(80.8)$

0.001

not done

$413(30.8)$

$358(34.0)$

$55(19.2)$

Patients with abnormalities among those who had foot screening at last visit, $n / n(\%)$ $324 / 926$

286/695 (41.2)

$38 / 231(16.5)$

0.001

No. of patients who have been on diabetes treatment at least 6 months in the project

179

mean fasting blood glucose of all patients on last visit (who have been on treatment at least 6 months)

$186(+/-$

101)

$187(+/-103)$

$182(+/-93)$

mean systolic blood pressure on last visit of all patients (who have been on treatment at least 6 months)

$135(+/-$

$135(+/-27)$

$138(+/-28)$

mean diastolic blood pressure on last visit of all patients (who have been on treatment at least 6 months)

$84(+/-15)$

$84(+/-15)$

$85(+/-15)$

0.82

mean difference in systolic blood pressure between first visit and last visit (of patients on treatment at least 6 months)

mean difference in diastolic blood pressure between first visit and last visit (of patients on treatment at least 6 months)

$0.51(+/-$

25.7) $\quad 1.8(+/-25.2) \quad-4.8(+/-27) \quad 0.38$

mean difference in fasting blood glucose between first visit and last visit (of patients on treatment at least 6 months)

$-1.4(+/-$
$15.2)$

$-0.9(+/-15.2)$

$-3.2(+/-14.7)$

0.44
BMI was 25.3 (IQR 21.5-29.8) and 88.1\% of patients had type 2 diabetes; $94 \%$ received HIV testing of whom $3.8 \%$ were HIV positive. At the time of enrolment, $53.8 \%$ of patients had pre-existing hypertension that had been present for a median period of 25 months (IQR 7-82). While the prevalence of known hypertension was not significantly different between patients in district hospitals and patients in health centres, the latter had significantly higher median blood pressure and higher prevalence of raised blood pressure. There were no significant differences in age, sex, type of diabetes, median FBG, median BMI and HIV status between patients attending District Hospitals and Health Centres at baseline. Significant differences in smoking, use of alcohol, BMI categories and diabetes control were caused by large differences in missing data between district hospitals and health centres.

\section{Quality of care and complications (Table 2)}

Patients were followed in the pilot for a median duration of 8.8 months. Follow up time was significantly longer for patients attending district hospitals (9.5 months vs. 7.2 months; $\mathrm{p}=0.001)$. Retention in care was $73.3 \%$, with no difference between hospitals and health centres.

There were no differences between patients at hospitals and health centres in HIV test coverage (93.6\%), weight measurements $(83.5 \%)$ or BP measurements $(92.4 \%)$ recorded on the NCD master-card. Blood glucose was recorded in $92.5 \%$ of patients at their last visit with a significantly higher proportion done among patients in hospitals $(95.8 \%$ vs. $80.4 \%$; $=0.001)$.

Of patients who had been on anti-diabetic treatment for at least 6 months, mean FBG on the last visit was $186 \mathrm{mg} / \mathrm{dL}$ (+/- 101), representing a mean decrease in FBG between enrolment and the last visit of $19 \mathrm{mg} / \mathrm{dL}(+/-122)$, with no significant differences between hospitals and health centres. Foot examination was done among $69.2 \%$ of patients during their last visit; with a higher proportion done at health centres compared to hospitals $(80.8 \%$ vs $66.0 \%$; $=0.001)$. Of patients who had foot screening, $35.0 \%$ had either ulcers, deformities or vascular disease, with a higher proportion reported at hospitals compared to health centres $(41.2 \%$ vs $16.5 \% ; \mathrm{p}=0.001)$.

Only $30.1 \%$ of patients received any retinal screening during the study period, with a higher proportion reported at hospitals compared to health centres $(37.0 \%$ vs $4.5 \%$; $\mathrm{p}=0.001)$. Of those screened, $15.6 \%$ had findings suggesting diabetic retinopathy. The proportion of patients who had urine testing was very low $(0.9 \%)$, with a significantly higher proportion done in health centres compared to hospitals $(3.5 \%$ vs $0.2 \% ; p=0.001)$. 
Table 3. Factors associated with Diabetes control and with Blood Pressure control

\begin{tabular}{|c|c|c|c|c|c|c|c|c|c|c|c|c|}
\hline & \multicolumn{6}{|c|}{$\begin{array}{c}\text { DM control } \\
\text { (fasting blood glucose reading (FBS) } \leq 130 \text { in last visit) }{ }^{*}\end{array}$} & \multicolumn{6}{|c|}{$\begin{array}{c}\text { BP control } \\
\text { (BP reading systolic }<140 \text { AND diastolic }<90 \text { ) in last visit** }\end{array}$} \\
\hline & \multirow[t]{2}{*}{$n / n$} & \multirow[t]{2}{*}{$\%$} & \multicolumn{2}{|c|}{$\begin{array}{l}\text { Univariate } \\
\text { (unadjusted) }\end{array}$} & \multicolumn{2}{|c|}{$\begin{array}{l}\text { Multivariate } \\
\text { (adjusted) }\end{array}$} & \multirow[t]{2}{*}{$\mathrm{n} / \mathrm{n}$} & \multirow[t]{2}{*}{$\%$} & \multicolumn{2}{|c|}{$\begin{array}{l}\text { Univariate } \\
\text { (unadjusted) }\end{array}$} & \multicolumn{2}{|c|}{$\begin{array}{l}\text { Multivariate } \\
\text { (adjusted) }\end{array}$} \\
\hline & & & $\begin{array}{c}\mathrm{OR} \\
(95 \% \mathrm{Cl})\end{array}$ & $p$-value & $\begin{array}{c}\mathrm{aOR} \\
(95 \% \mathrm{Cl})^{\mathrm{s}}\end{array}$ & $p$-value & & & $\begin{array}{c}\mathrm{OR} \\
(95 \% \mathrm{Cl})\end{array}$ & $p$-value & $\begin{array}{c}\mathrm{aOR} \\
(95 \% \mathrm{Cl})^{\$}\end{array}$ & $p$-value \\
\hline & $437 / 1231$ & 35.5 & & & & & $631 / 1237$ & 47.1 & & & & \\
\hline \multicolumn{13}{|l|}{ Health Facility } \\
\hline $\mathrm{DH}$ & $350 / 1001$ & 35 & ref & & & & $502 / 978$ & 51.3 & ref & & & \\
\hline $\mathrm{HC}$ & $87 / 230$ & 37.8 & $\begin{array}{c}0.8(0.6- \\
1.2)\end{array}$ & 0.41 & & NS & $129 / 259$ & 49.8 & $\begin{array}{l}0.9(0.7- \\
1.2)\end{array}$ & 0.66 & & NS \\
\hline Age (in years) & & & $\begin{array}{l}1.0(0.9- \\
1.1)\end{array}$ & 0.75 & & NS & & & $\begin{array}{c}0.9(0.9- \\
1.0)\end{array}$ & 0.0001 & $\begin{array}{l}0.9(0.9- \\
1.0)\end{array}$ & 0.01 \\
\hline \multicolumn{13}{|l|}{ Gender } \\
\hline Male & $205 / 502$ & 40.8 & ref & & & & $271 / 507$ & 53.5 & ref & & & \\
\hline Female & $232 / 729$ & 31.8 & $\begin{array}{l}0.7(0.5- \\
0.8)\end{array}$ & 0.001 & $\begin{array}{l}0.7(0.5- \\
0.9)\end{array}$ & 0.02 & $360 / 730$ & 49.3 & $\begin{array}{l}0.8(0.7- \\
1.1)\end{array}$ & 0.15 & & NS \\
\hline \multicolumn{13}{|l|}{ Diabetes Type } \\
\hline Type 1 & $58 / 141$ & 41.1 & ref & & & & $100 / 131$ & 76.3 & ref & & & \\
\hline Type 2 & $375 / 1086$ & 34.5 & $\begin{array}{l}0.8(0.5- \\
1.1)\end{array}$ & 0.12 & & NS & $531 / 1102$ & 48.2 & $\begin{array}{l}0.3(0.2- \\
0.4)\end{array}$ & 0.001 & & NS \\
\hline Missing & $4 / 4$ & 100 & & & & & $0 / 4$ & 0 & & & & \\
\hline $\begin{array}{l}\text { Duration in care } \\
\text { (months) }^{\&}\end{array}$ & & & $\begin{array}{l}1.0(0.9- \\
1.1)\end{array}$ & 0.53 & & NS & & & $\begin{array}{l}1.0(1.0- \\
1.0)\end{array}$ & 0.49 & & NS \\
\hline \multicolumn{13}{|l|}{ BMI } \\
\hline $\begin{array}{l}\text { Underweight }(< \\
\text { 18.5) }\end{array}$ & $24 / 93$ & 25.8 & $\begin{array}{l}0.6(0.4- \\
1.0)\end{array}$ & 0.06 & & NS & $63 / 86$ & 73.3 & $\begin{array}{l}1.9(1.1- \\
3.2)\end{array}$ & 0.02 & $\begin{array}{l}1.9(1.0- \\
3.4)\end{array}$ & 0.04 \\
\hline $\begin{array}{l}\text { Normal weight } \\
\text { (18.5 to } 24.9)\end{array}$ & $125 / 343$ & 36.4 & ref & & & & $209 / 355$ & 58.9 & ref & & ref & \\
\hline $\begin{array}{l}\text { Overweight }(25 \text { to } \\
29.9)\end{array}$ & $100 / 267$ & 37.5 & $\begin{array}{l}1.0(0.8- \\
1.5)\end{array}$ & 0.80 & & NS & $134 / 271$ & 49.4 & $\begin{array}{l}0.7(0.5- \\
0.9)\end{array}$ & 0.02 & $\begin{array}{l}0.7(0.5- \\
1.0)\end{array}$ & 0.09 \\
\hline Obese $(\geq 30)$ & $85 / 239$ & 35.6 & $\begin{array}{l}1.0(0.7- \\
1.4)\end{array}$ & 0.80 & & NS & $84 / 241$ & 34.9 & $\begin{array}{c}0.4(0.3- \\
0.5)\end{array}$ & 0.001 & $\begin{array}{c}0.4(0.3- \\
0.6)\end{array}$ & 0.001 \\
\hline Missing & $103 / 289$ & 35.6 & $\begin{array}{c}1.0(0.7- \\
1.3)\end{array}$ & 0.80 & & NS & $141 / 284$ & 49.6 & $\begin{array}{c}0.7(0.5- \\
0.9)\end{array}$ & 0.02 & $\begin{array}{c}0.8(0.6- \\
1.1)\end{array}$ & 0.22 \\
\hline \multicolumn{13}{|l|}{ HIV status } \\
\hline Unknown & $27 / 78$ & 34.6 & $\begin{array}{c}0.9(0.6- \\
1.5)\end{array}$ & 0.80 & & NS & $30 / 75$ & 40 & $\begin{array}{c}0.6(0.4- \\
1.0)\end{array}$ & 0.07 & & NS \\
\hline & & & 0.5 (0.3- & & & NS & & & $2.0(1.1-$ & & & \\
\hline Positive & $11 / 47$ & 23.4 & 1.1) & 0.08 & & & $32 / 47$ & 68.1 & $3.8)$ & 0.03 & & NS \\
\hline Negative & $399 / 1106$ & 36.1 & ref & & & & $569 / 1115$ & 51 & ref & & & \\
\hline \multicolumn{13}{|l|}{ * data missing, } \\
\hline \multicolumn{13}{|l|}{$n=108$} \\
\hline \multicolumn{13}{|l|}{ ** data missing, } \\
\hline \multicolumn{13}{|l|}{$n=102$} \\
\hline \multicolumn{13}{|c|}{ \$adjusted for all other variables in the model; significant associations reported only (NS=not significant) } \\
\hline${ }^{\&}$ Months in care fre & first to last & durin & the reporti & & & & & & & & & \\
\hline
\end{tabular}


Factors associated with diabetes and blood pressure control (Table 3)

At the end of the project period, adequate diabetes control was achieved by $35.5 \%$ of all patients, with no significant difference between those receiving care in hospitals or health centres $(35.0 \%$ vs. $37.8 \%$; $=0.41)$. In multivariate logistic regression analysis, only sex was independently associated with diabetes control: females were less likely to achieve adequate glucose levels (aOR $0.7 ; 95 \% \mathrm{CI}$ 0.5-0.7) when adjusted for health facility type, age, diabetes type, duration in care, BMI and HIV status.

Adequate BP control was achieved by $47.1 \%$ of all patients, with no significant difference between those receiving care in hospitals or health centres ( $51.3 \%$ vs. $49.8 \%$; $\mathrm{p}=0.66)$

In multivariate logistic regression analysis, age and BMI were independently associated with achieving BP control. Increasing age (aOR 0.9; 95\% CI: 0.9-1.0) and being underweight $(\mathrm{BMI}<18.5$ : aOR 1.9; 95\% CI 1.0-3.4) or obese (BMI>30: aOR 0.4; 95\% CI: 0.3-0.6) were associated with a decreased likelihood of achieving BP control when adjusted for health facility type, sex, diabetes type, duration in care, BMI and HIV status.

\section{Discussion}

We demonstrated that decentralizing diabetes care from district hospitals to health centres was possible using a mentorship approach. Retention measured over only 12 months was similar in district hospitals and health centres. Our findings revealed no differences in glucose or BP control in patients managed by nurses or medical assistants at health centres compared to those managed by clinical officers in hospitals. Other studies from the region showed similar outcomes. In a nurse-managed community-based hypertension program in Ghana, $72 \%$ of patients were retained in care at 12 months with good $\mathrm{BP}$ control $^{6}$. In a rural setting in South Africa, nurses were able to successfully manage patients with hypertension and diabetes ${ }^{7,8,9}$. In nurse led diabetes clinics at health centres and hospitals in Rwanda, nurses were reported to have high levels of adherence to treatment protocols and successful treatment outcomes after two years of follow-up ${ }^{10,11}$.

Our study had several limitations; we used FBG instead of HBA1C and assessed control based on a single (last) measurement We thus were unable to assess glucose control over time. We only had one size of BP cuff which may have produced inaccurate readings in patients with very low or high BMI.

By the end of the intervention period, 21\% of patients chose to receive their diabetes care at a health centre, rather than at a hospital. This was similar to our initial estimate of $25 \%$ of diabetes patients likely to choose services offered closer as they lived $80 \mathrm{~km}$ or more away from the hospital. Other rural settings in Africa have shown variable use of decentralized health centre services for diabetes. In rural South Africa, $79 \%$ of patients with NCDs, including diabetes, transferred care from the hospital to health centres ${ }^{7}$, whilst in rural Ethiopia only $11 \%$ of patients with diabetes transferred from a hospital to a health centre ${ }^{12}$.

Our retention rate of $73 \%$ compares favorably to other settings but our follow-up was limited to less than one year on average. Defaulting or loss-to-care is a significant challenge in many NCD programs in Africa. In Soweto, $47 \%$ of diabetic patients were lost from NCD focused care services within two years ${ }^{13}$. A hypertension program in Ghana had a retention rate of less than $30 \%$ at 12 months $^{6}$. In contrast, in Rwanda where mentoring was conducted at health centre diabetes clinics, only $17 \%$ of diabetic patients were lost to follow-up after 24 months ${ }^{11}$.

In our cohort, levels of glucose and BP control were poor (36\% and $47 \%$, respectively and not significantly different between District Hospitals and Helath Centres). Other studies in Africa have reported similar poor levels of glucoseand BP control amongst diabetics enrolled in care ${ }^{14,15,16}$.

Quality of care was measured by completeness of measurements and screening for feet abnormalities, proteinuria and retinopathy. Routine measurements of BP and glucose were relatively easy to achieve and similar to rates reported in rural Rwanda (BP 96\%, glucose 93\% ${ }^{10}$ ). Rates of foot screening $(69 \%)$ although incomplete was also comparable to rural Rwanda $(72 \%)^{10}$ but higher than previously reported in rural Malawi $(17 \%)^{17}$. Only $30 \%$ of patients received fundoscopy in our cohort. However, most patients receiving care at health centres needed referral to hospital (where clinical officers trained in fundoscopy are based) for retinal screening, a step which very few managed to take. Monitoring of complications that require additional pathways such as retinal and urine screening are especially challenging to achieve. Monitoring of diabetic complications with methodologies appropriate for health centers and with a functioning referral system for severe organ disease require urgent investments, including in human resources and (innovative) diagnostic equipment.

One of the features of our pilot was the introduction of new clinic-based NCD master-cards. These cards were well accepted as they were based on the widely used ART mastercard. Their use required the training and presence of an NCD-clinic clerk, a lay cadre allocated to this duty on clinic days. Incomplete documentation, especially of baseline complications was challenging and required continuous attention. The introduction of protocols and standardized clinical forms have been used in other settings with variable success. The introduction of structured clinical records for hypertension and diabetes in primary health care clinics in Cape Town had no effect on glucose or BP control ${ }^{18}$. In Soweto, a "traffic light system" was successful at identifying patients needing referral, but less successful for patients needing closer monitoring or yearly screening ${ }^{13}$. The use of an integrated clinical tool combined with educational outreach to nurses in health centres in South Africa, did not lead to an intensification of treatment in patients with hypertension or diabetes compared to facilities who did not use the tool ${ }^{19}$.

We believe that in our pilot, the on-site mentorship, rather than the new record system brought improvement. The introduction of the master-card alone, before the mentoring visits resulted in poor documentation. Training without mentorship is thought to be insufficient to improve quality of care $^{20}$. The HIV program in Malawi has used clinical mentorship as an effective way to ensure that training information is put into practice, leading to improved quality of care $^{21}$.

A key part of this intervention was the involvement of expert people living with diabetes giving patient education. Patient education is often lacking in overstretched primary health care clinics. A study assessing quality of care in 75 diabetic patients in rural Malawi found that $40 \%$ of patients had 
no information about diabetes ${ }^{17}$. At health centres in Cape Town, patients with diabetes had little understanding of how to manage hyper- or hypoglycaemia and poor knowledge of the importance of good blood glucose control ${ }^{15}$. The utilization of "expert patients" was advocated for in the UK in 2002 and was later used successfully in a program in rural South Africa ${ }^{22,23}$. Similar to our experience in Malawi, leaders such as school teachers, nurses or community health workers living with diabetes were selected and trained in the use of a "Zakhe" (his/hers) diabetes programme that included use of pictorial flip charts ${ }^{8}$. The DAM was formed in 2007 as a channel for health awareness about diabetes and patient advocacy. Using EDCs from this association strengthened the relationship between the association and patients and enabled DAM members to play a new role in the health system.

\section{Conclusion}

Decentralisation of diabetes care to health centres showed satisfactory uptake and short-term retention. EDCs played an important role in health education, peer support and small administrative tasks. Quality of care as measured by diabetes and BP control and consistency of screening for complications did not differ between hospitals and health centres, but was insufficient at both levels. A higher priority for delivering quality diabetes care at all levels of the health system is needed, and especially at peripheral health centres, given the large affected population residing in rural areas.

\section{Declarations}

\section{Ethics approval and consent to particiapate}

Ethical approval was obtained by the College of Medicine Research and Ethics Committee (P.09/18/2470) and we obtained prior support from district health offices. As a retrospective audit of routinely collected, standard service delivery data that had been fully anonymized before analysis, we were exempted from obtaining individual informed consent.

\section{Availability of data and materials}

The datasets used and/or analysed during the current study are available from the corresponding author on reasonable request.

\section{Competing interests}

The authors declare that they have no competing interests.

\section{Funding}

This project was made possible by the financial support of World Diabetes Foundation.

\section{Authors' contributions}

CP, JVO, JT and AA conceived the project. CP, TA , CM and GM led the guideline and training curriculum development process. TA, JM, SN and MJ gave technical support on guideline and curriculum development. AA, MVL, GK and SK led data analysis. CP, MVL and JVO led manuscript writing. All authors contributed to and approved the final manuscript.

\section{Acknowledgements}

We would like to acknowledge the support of the managers and staff in the Malawi Ministry of Health who made this possible.

\section{References}

1. Mathers CD, Loncar D. Projections of global mortality and burden of disease from 2002 to 2030. PLoS Med. 2006. doi:10.1371/journal. pmed.0030442

2. Price AJ, Crampin AC, Amberbir A, et al. Prevalence of obesity, hypertension, and diabetes, and cascade of care in sub-Saharan Africa: a cross-sectional, population-based study in rural and urban Malawi. Lancet Diabetes Endocrinol. 2018. doi:10.1016/S2213-8587(17)304321

3. Wood R, Viljoen V, Van Der Merwe L, Mash R. Quality of care for patients with non-communicable diseases in the Dedza District, Malawi. African J Prim Heal Care Fam Med. 2015. doi:10.4102/phcfm.v7i1.838

4. Pfaff C, Scott V, Hoffman R, Mwagomba B. You can treat my HIV - But can you treat my blood pressure? Availability of integrated HIV and noncommunicable disease care in northern Malawi. African J Prim Heal Care Fam Med. 2017. doi:10.4102/phcfm.v9i1.1151

5. Chikowe I, Mwapasa V, Kengne AP. Analysis of rural health centres preparedness for the management of diabetic patients in Malawi. BMC Res Notes. 2018. doi:10.1186/s13104-018-3369-7

6. Adler AJ, Laar A, Prieto-Merino D, et al. Can a nurse-led community-based model of hypertension care improve hypertension control in Ghana? Results from the ComHIP cohort study. BMJ Open. 2019;9(4):1-10. doi:10.1136/bmjopen-2018-026799

7. Coleman R, Gill G, Wilkinson D. Noncommunicable disease management in resource-poor settings: a primary care model from rural South Africa. Bull World Health Organ. 1998;76(6):633-640. http://www.ncbi.nlm.nih.gov/pubmed/10191559\%0Ahttp://www. pubmedcentral.nih.gov/articlerender.fcgi?artid=PMC2312489.

8. Gill G V., Price C, Shandu D, Dedicoat M, Wilkinson D. An effective system of nurse-led diabetes care in rural Africa. Diabet Med. 2008. doi:10.1111/j.1464-5491.2008.02421.x

9. Price C, Shandu D, Dedicoat M, Wilkinson D, Gill G V. Long-term glycaemic outcome of structured nurse-led diabetes care in rural Africa. Qjm. 2011;104(7):571-574. doi:10.1093/qjmed/hcr005

10. Ndayisaba A, Harerimana E, Borg R, et al. A Clinical Mentorship and Quality Improvement Program to Support Health Center Nurses Manage Type 2 Diabetes in Rural Rwanda. J Diabetes Res. 2017. doi: $10.1155 / 2017 / 2657820$

11. Tapela N. Diabetes in Rural Rwanda: High Retention and Positive Outcomes after 24 Months of Follow-up in the Setting of Chronic Care Integration. Int J Diabetes Clin Res. 2017. doi:10.23937/2377$3634 / 1410058$

12. Mamo Y, Seid E, Adams S, Gardiner A, Parry E. A primary healthcare approach to the management of chronic disease in Ethiopia: An example for other countries. Clin Med J R Coll Physicians London. 2007. doi:10.7861/clinmedicine.7-3-228

13. Katz I, Schneider H, Shezi Z, et al. Managing type 2 diabetes in Soweto-The South African Chronic Disease Outreach Program experience. Prim Care Diabetes. 2009. doi:10.1016/j.pcd.2009.06.007

14. Cohen DB, Allain TJ, Glover S, et al. A survey of the management, control, and complications of diabetes mellitus in patients attending a diabetes clinic in Blantyre, Malawi, an area of high HIV prevalence. Am J Trop Med Hyg. 2010. doi:10.4269/ajtmh.2010.10-0104

15. Steyn K, Levitt NS, Patel M, et al. Hypertension and diabetes: Poor care for patients at community health centres. South African Med J. 2008.

16. Levitt NS, Bradshaw D, Zwarenstein MF, Bawa AA, Maphumolo S. Audit of public sector primary diabetes care in Cape Town, South Africa: High prevalence of complications, uncontrolled hyperglycaemia, and hypertension. Diabet Med. 1997. doi:10.1002/(SICI)10969136(199712)14:12<1073::AID-DIA498>3.0.CO;2-9

17. Assayed AA, Muula AS, Nyirenda MJ. The quality of care of 
diabetic patients in rural Malawi: A case of Mangochi district. Malawi Med J. 2014.

18. Steyn K, Lombard C, Nomonde Gwebushe, et al. Implementation of national guidelines, incorporated within structured diabetes and hypertension records at primary level care in Cape Town, South Africa: A randomised controlled trial. Glob Health Action. 2013;6(1). doi:10.3402/gha.v6i0.20796

19. Fairall LR, Folb N, Timmerman V, et al. Educational Outreach with an Integrated Clinical Tool for Nurse-Led Non-communicable Chronic Disease Management in Primary Care in South Africa: A Pragmatic Cluster Randomised Controlled Trial. PLoS Med. 2016. doi:10.1371/ journal.pmed.1002178

20. Leslie HH, Gage A, Nsona H, Hirschhorn LR, Kruk ME. Training and supervision did not meaningfully improve quality of care for pregnant women or sick children in sub-Saharan Africa. Health Aff. 2016. doi:10.1377/hlthaff.2016.0261
21. Chien E, Phiri K, Schooley A, Chivwala M, Hamilton J, Hoffman RM. Successes and challenges of HIV mentoring in Malawi: The mentee perspective. PLoS One. 2016. doi:10.1371/journal.pone.0158258

22. Tattersall R. The expert patient: A new approach to chronic disease management for the twenty-first century. Clin Med (Northfield Il). 2002. doi:10.7861/clinmedicine.2-3-227

23. Price C, Shandu D, Gill G V. Diabetes education and empowerment: Lessons from rural South Africa. Pract Diabetes Int. 2007. doi:10.1002/ pdi. 1101 